\title{
Synthesis and Fluorescence Property of Mn-Doped ZnSe Nanowires
}

\author{
Dongmei Han, ${ }^{1,2}$ Chunfeng Song, ${ }^{1,3}$ and Xiaoyu $\mathrm{Li}^{1,3}$ \\ ${ }^{1}$ School of Materials Science and Engineering, Beijing University of Chemical Technology, Beijing 100029, China \\ ${ }^{2}$ State Key Laboratory of Chemical Resource Engineering, Beijing University of Chemical Technology, Beijing 100029, China \\ ${ }^{3}$ Key Laboratory for Nanomaterials, Beijing University of Chemical Technology, Ministry of Education, Beijing 100029, China
}

Correspondence should be addressed to Xiaoyu Li, lixy@mail.buct.edu.cn

Received 1 November 2009; Revised 27 April 2010; Accepted 27 May 2010

Academic Editor: Raymond Whitby

Copyright (C) 2010 Dongmei Han et al. This is an open access article distributed under the Creative Commons Attribution License, which permits unrestricted use, distribution, and reproduction in any medium, provided the original work is properly cited.

\begin{abstract}
Water-soluble Mn-doped ZnSe luminescent nanowires were successfully prepared by hydrothermal method without any heavy metal ions and toxic reagents. The morphology, composition, and property of the products were investigated. The experimental results showed that the Mn-doped ZnSe nanowires were single well crystallized and had a zinc blende structure. The average length of the nanowires was about $2-3 \mu \mathrm{m}$, and the diameter was $80 \mathrm{~nm}$. With the increase of $\mathrm{Mn}^{2+}$-doped concentration, the absorbance peak showed large difference. The UV-vis absorbance spectrum showed that the Mn-doped ZnSe nanowires had a sharp absorption band appearing at $360 \mathrm{~nm}$. The PL spectrum revealed that the nanowires had two distinct emission bands centered at 432 and $580 \mathrm{~nm}$.
\end{abstract}

\section{Introduction}

As biomedical labeling reagents, high-quality colloidal luminescent semiconductor nanocrystals, such as CdSe, have attracted much attention because of their apparent, narrow, and symmetric photoluminescence, broad and intense absorption band, and tunable emission peak positions $[1,2]$. However, many results indicate that any leakage of cadmium from the nanocrystals would be toxic and fatal to biological systems. Some attempts have been made to develop noncadmium materials. Transition metal ion-doped quantum dots (d-dots) can meet this requirement. The sp-d exchange interaction between the semiconductor and the transition metal ions induces unique optoelectronic properties [3]; Mn-doped II-VI semiconductors arouse general interest in particular, [4-6]. As a wide-band-gap material, ZnSe is suitable for the fabrication of various optoelectronic devices, biolabeling, and hosts for the formation of doped nanocrystal [7-9]. Recent researches have been carried out to explore for the synthesis of transition metal ion-doped $\mathrm{ZnSe}$ nanocrystals such as $\mathrm{Cu}^{-}, \mathrm{Mn}^{-}$, and $\mathrm{Co}$-doped $\mathrm{ZnSe}$ nanomaterials. [10-12]

At present, the main method of preparing transition metal ion-doped ZnSe nanomaterials is colloid chemistry route. The experiments are typically carried out with high temperature, toxic, and explosive chemical reagents. In particular, as-prepared products cannot be directly dispersed in the water phase due to their hydrophobic surfaces. A further reaction is needed to achieve the phase transfer for the aqueous environment. More recently, Nie and his coworkers [13] developed an alternative method to synthesize nanocrystals in aqueous solution. On the basis of those and other encouraging results $[14,15]$, we recently focused on the possibilities to synthesize metal ion-doped ZnSe directly in aqueous solution and explored the impact of system on morphology and performance of the nanomaterials.

In this paper, we report on the preparation of highcrystalline Mn-doped ZnSe nanowires (NWs) by a simple method, solution synthetic methodology using $\alpha$ cyclodextrins as stabilizer, and investigated the relationship between the structures and the properties.

\section{Experimental}

All chemicals and solvents in our work were commercial products used as received without further purification. $\alpha$ cyclodextrin $(\alpha-\mathrm{CD})$ was a product of Alfa Company, selenium powder (99.95\%) was obtained from Beijing Reagent 
Company. Zinc acetate $\left(\mathrm{Zn}(\mathrm{OAc})_{2} \cdot 2 \mathrm{H}_{2} \mathrm{O}\right)$, manganese acetate $\left(\mathrm{Mn}(\mathrm{OAc})_{2} \cdot 4 \mathrm{H}_{2} \mathrm{O}\right)$, sodium sulfite, sodium hydroxide $(\mathrm{NaOH})$, sodium citrate, and anhydrous ethanol were analytical grade and purchased from Beijing Reagent Company. Deionized water was used throughout all experiments.

The synthesis of $\mathrm{ZnSe}$ and $\mathrm{Mn}$-doped $\mathrm{ZnSe}$ nanowires was adapted from a procedure developed for CdSe nanoparticles [16]. A brief description is provided as follows. A total of $0.2588 \mathrm{~g}$ of sodium citrate, $10 \mathrm{~mL}$ of $0.04 \mathrm{M} \mathrm{Zn}(\mathrm{OAc})_{2}$ solution and $0.08 \mathrm{mmol} \mathrm{Mn}(\mathrm{OAc})_{2}$ were added to $225 \mathrm{~mL}$ of deionized water. Under the magnetic agitation, $2 \mathrm{~g}$ of $\alpha-\mathrm{CD}$ was introduced to the flask. After the $\mathrm{pH}$ value was adjusted to 10 with $1 \mathrm{M} \mathrm{NaOH}$ solution, the mixture was purged with $\mathrm{N}_{2}$ for $30 \mathrm{~min}$. Then $6 \mathrm{~mL}$ of $0.05 \mathrm{M} \mathrm{Na}_{2} \mathrm{SeSO}_{3}$ solution was added. When mixed uniformly, the solution was transferred to the vessel. It was laid to the oven with temperature $180^{\circ} \mathrm{C}$ for 4 days. After being naturally cooled to the room temperature, the mixture was centrifuged at $5000 \mathrm{rpm} / \mathrm{min}$ for 10 minutes and washed several times by deionized water and absolute ethanol. Having dried in vacuum at $80^{\circ} \mathrm{C}$ for 3 hours, the yellow products were obtained.

The transmission electron microscope (TEM) images were taken by $\mathrm{H}-800$ microscope with an acceleration voltage of $80 \mathrm{kV}$. The high-resolution TEM images were obtained by employing a JEOL 3010 microscopy with a $300 \mathrm{kV}$ accelerating voltage. Samples for TEM were prepared by placing a drop of the sample suspension on a copper grid coated with carbon and were allowed to dry in air. The samples were characterized by X-ray diffraction (XRD), with scanning rate $10^{\circ} / \mathrm{min}$, on $\mathrm{D} / \mathrm{max} 2500 \mathrm{VB} 2+/ \mathrm{PC} \mathrm{X}-$ ray diffractometer using graphite monochromatized $\mathrm{Cu} \mathrm{K} \alpha$ radiation $(\lambda=1.5406 \AA)$. The UV-vis absorbance spectra were obtained on a Shimadzu UV-3150 spectrophotometer in the range of $200 \sim 800 \mathrm{~nm}$ at room temperature. Optical densities were kept within 0.2 at the excitation wavelength. The photoluminescence (PL) spectra of the as-prepared Mndoped $\mathrm{ZnSe}$ nanomaterials were measured using a Varian Cary-Eclipse fluorophotometer.

\section{Results and Discussion}

The XRD patterns of the ZnSe and Mn-doped ZnSe NWs are displayed in Figure 1. The XRD peaks of the undoped ZnSe NWs can be well indexed to zinc blende ZnSe crystals (JCPDS Card No. 37-1463). In the case of Mn-doped ZnSe NWs synthesized under the same conditions, the peaks are also indexed to zinc blende ZnSe. However, the main peaks of (111), (220), and (311) planes move $0.19^{\circ}$ to the left because $\mathrm{Mn}$ was implanted to $\mathrm{ZnSe}$ internal lattice. The peak intensities at $2 \theta$ of $\leftarrow 31.0^{\circ}[(200)$ plane $]$ and $\leftarrow 57.0^{\circ}[(222)$ plane] are markedly increased, which indicates that these Mn-doped nanowires have more advantages on growth along the two directions. The peaks which are observed at $\leftarrow 38.0^{\circ}$ and $49.5^{\circ}$ match those of wurtzite phase [(102) plane] and [(103) plane] because the synthesis process is affected by the introduction of impurity ions $\mathrm{Mn}^{2+}$ into the host lattice which can significantly affect the reaction rate, which is reflected by a reduction in the crystal phase [17].

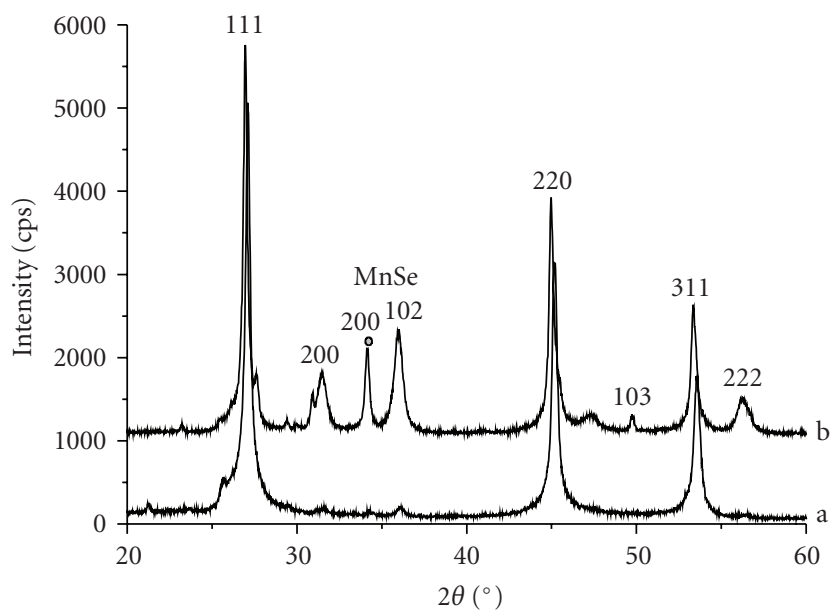

Figure 1: XRD patterns of the ZnSe (a) and Mn-doped ZnSe (b) nanowires.

It is similar to the characters of the Mn-ZnSe nanoparticles reported by Mahamuni and coworkers [18]. A similar effect has also been reported for $\mathrm{Mn}^{2+}$-doped $\mathrm{ZnSe}$ nanowires prepared using the high-temperature organometallic synthesis route [19]. The peak at $\leftarrow 34.0^{\circ}$ is observed that matches those of MnSe [(200) plane] which indicates that there may be a small amount of MnSe produced.

Figure 2 shows the TEM images for Mn-doped $\mathrm{ZnSe}$ nanowires. The average length is about 2-3 $\mu \mathrm{m}$. It reveals that they are all straight and have a smooth surface without any nanoparticle impurities. Their average diameter is $80 \mathrm{~nm}$. This result is different from other cases. Colloidal Mn-doped ZnSe nanomaterials prepared in the solvent of the typical TOP/ HDA/TOPO system are generally particles with the size of several nanometers. In an improved method, nanowires are obtained mostly with the diameter of several nanometers and length up to $200 \mathrm{~nm}$ [17].

The high-resolution TEM image of the nanowires and corresponding fast Fourier transformed electron diffraction (FFT ED) pattern are displayed, respectively. From the further analysis, it was found that the growths of crystal lattice are clear, even though the nanowires are very thick. The selected area ED pattern, consisted of discrete and bright spots, indicating that the nanowires are all typically single well-crystallized diffraction pattern of ZnSe. The ED pattern confirms that the zinc blende-structured ZnSe nanowires grow along the (200) direction. The image shows that the highly crystalline (200) planes, separated by $2.819 \AA$, are close to that of cubic structured $\mathrm{ZnSe}$ crystal $2.834 \AA$.

UV-vis absorption spectra of Mn-doped ZnSe NWs with different $\mathrm{Mn}^{2+}$-doped concentrations are presented in Figure 3. An interesting pattern was revealed by this set of experiments. The absorption peaks of all samples are very strong. With the increase of $\mathrm{Mn}^{2+}$-doped concentration, the absorbance shows large difference. The two weak absorption peaks of 5\% Mn-doped ZnSe NWs emerge at $250 \mathrm{~nm}$ and $370 \mathrm{~nm}$. While $\mathrm{Mn}^{2+}$-doped concentration is increased to $10 \%$, the location of absorption peak is at $360 \mathrm{~nm}$ with stronger absorption intensity, but the absorption peaks are 


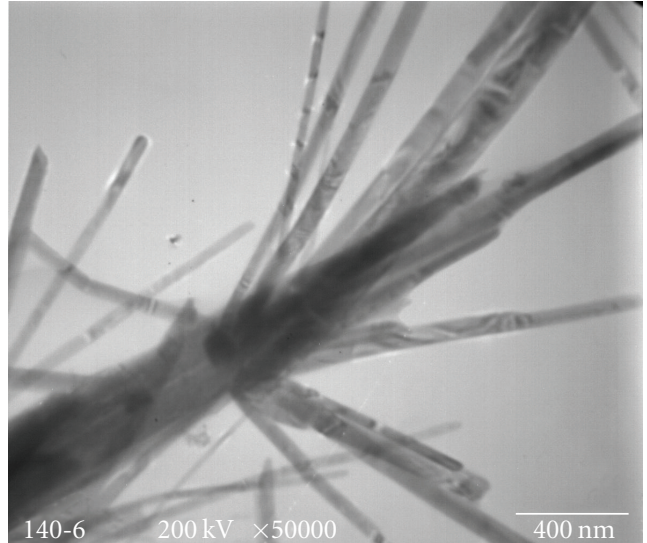

(a)

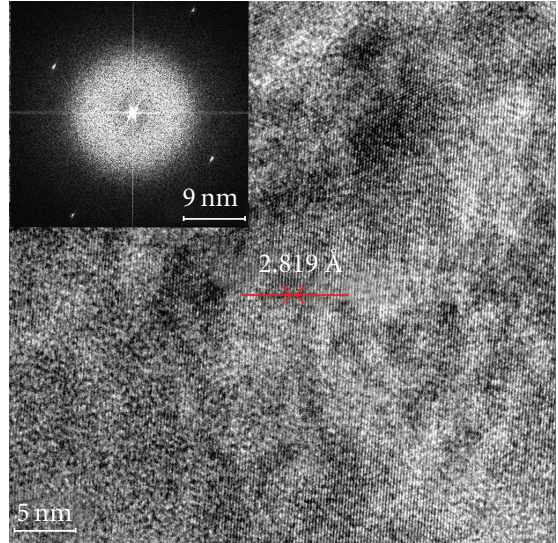

(b)

FIgure 2: TEM images of the Mn-doped ZnSe nanowires, HRTEM, and its SAED pattern images (inset).

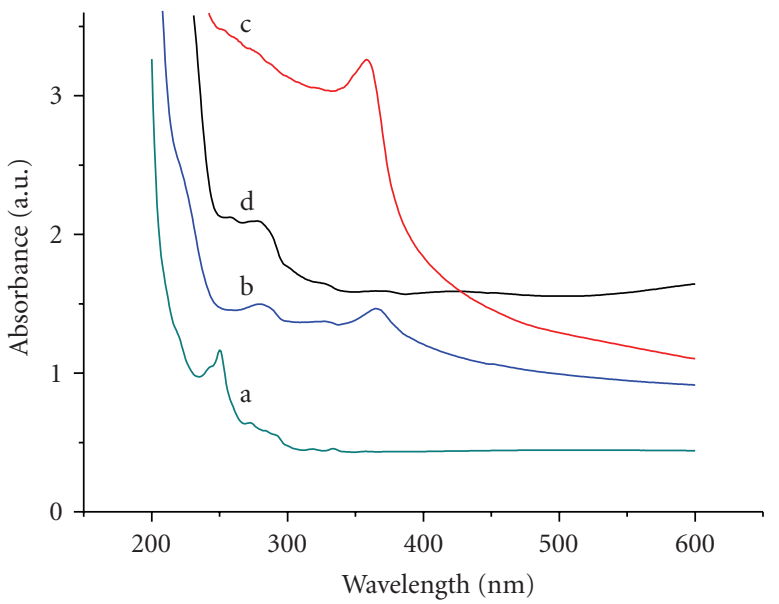

Figure 3: Absorption spectra of Mn-doped ZnSe with different $\mathrm{Mn}^{2+}$-doped concentrations (a: 0; b: 5\%; c: $10 \%$; d: 20\%).

similar with the pure ZnSe except of small red shift when the doped concentration is $20 \%$. It has been reported that the transition metal ion-doped semiconductor could form a doping energy level with the band gap of ZnSe [20]. The doping concentration could have an influence on optical properties to a large extent.

In our case, when doping concentration is low (5\%), the content of $\mathrm{Mn}$ is inadequate to reduce the band gap of $\mathrm{ZnSe}$ which results in $\mathrm{ZnSe}$ characteristic absorption and doped absorption emerging at the same time. The optimal doping concentration is $10 \%$. The dopant can serve to retard charge traps and effectively reduce the band gap within the suitable concentration. When the doping concentration is too high $(20 \%)$, the inconsistency of precipitation reaction may lead to precipitation separation and dopants failure to enter the internal lattice [20].

Figure 4 shows the optical absorption feature of Mndoped ZnSe NWs (10\% $\mathrm{Mn}^{2+}$-doped concentration). A

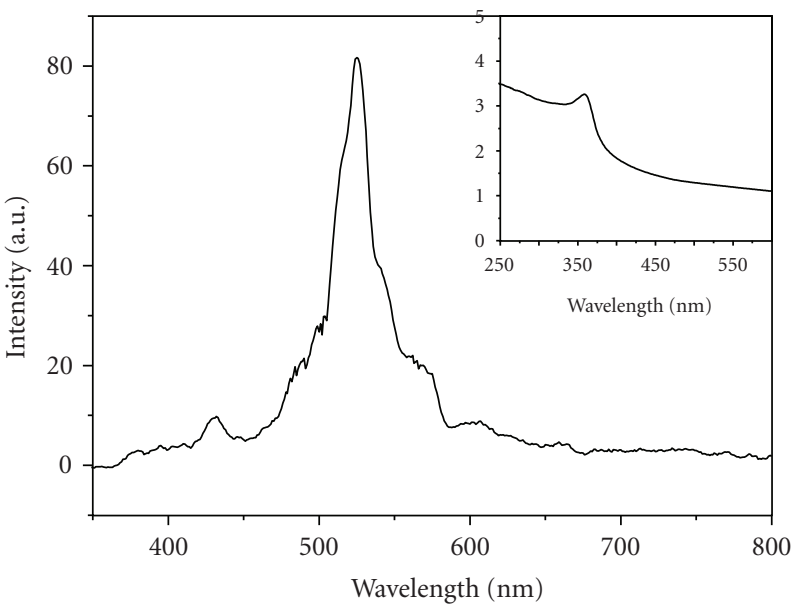

Figure 4: Photoluminescence spectrum and the absorption spectrum (inset) of Mn-doped $\mathrm{ZnSe}$.

sharp absorption band appears at $360 \mathrm{~nm}$. A red shift in the absorption band compared with pure $\mathrm{ZnSe}$ nanoparticles [14] suggests that the injected metal ions are isomorphic to $\mathrm{Zn}^{2+}$ ions and decrease the band gap. The corresponding luminescence spectrum with $350 \mathrm{~nm}$ excitation wavelength shows signs of two distinct emission bands centered at 432 and $540 \mathrm{~nm}$, respectively. These bands can be assigned to ZnSe trap state and $\mathrm{Mn}^{2+}{ }^{4} \mathrm{~T}_{1} \rightarrow{ }^{6} \mathrm{~A}_{1}$ emission. The halfpeak-wide of the highest emission peak is $29 \mathrm{~nm}$, which is very narrow and almost symmetrical.

Band-edge luminescence and $\mathrm{Mn}^{2+}$-related luminescence are competing processes. The absorption of a photon leads to the formation of an exciton. Luminescence results from recombination of the exciton as well as from the energy transfer to $\mathrm{Mn}^{2+} \mathrm{d}$-d levels. $\mathrm{Mn}^{2+}$ doping clearly leads the near-band-edge luminescence at $412 \mathrm{~nm}$ to the disappearance of, and the manganese orange emission band emerges at about $580 \mathrm{~nm}$ in Mn-doped ZnSe nanowires [18]. 


\section{Conclusion}

In summary, Mn-doped $\mathrm{ZnSe}$ nanowires were synthesized by hydrothermal method using $\alpha$-cyclodextrins as stabilizer. It was a convenient, efficient method to obtain the transition metal ion-doped one-dimensional $\mathrm{ZnSe}$ nanomaterial which can be directly dispersed in the water phase. The average length of nanowires was about 2-3 $\mu \mathrm{m}$, and the diameter was $80 \mathrm{~nm}$. Mn-doped ZnSe NWs were indexed to zinc blende ZnSe crystals. After being doped, the peak of the absorption spectra of the nanowires appeared at $360 \mathrm{~nm}$, and their emission band lied at about $580 \mathrm{~nm}$. This method is simple for synthesizing $\mathrm{Mn}$-doped $\mathrm{ZnSe}$ nanowires and provides a flexible approach for applications of biomedical labeling reagents.

\section{Acknowledgment}

This paper was financially supported by the National Natural Science Foundation of China (no. 50673008).

\section{References}

[1] X. Peng, M. C. Schlamp, A. V. Kadavanich, and A. P. Alivisatos, "Epitaxial growth of highly luminescent CdSe/CdS core/shell nanocrystals with photostability and electronic accessibility," Journal of the American Chemical Society, vol. 119, no. 30, pp. 7019-7029, 1997.

[2] B. O. Dabbousi, J. Rodriguez-Viejo, F. V. Mikulec et al., "(CdSe) $\mathrm{ZnS}$ core-shell quantum dots: synthesis and characterization of a size series of highly luminescent nanocrystallites," Journal of Physical Chemistry B, vol. 101, no. 46, pp. 94639475, 1997.

[3] B. Steitz, Y. Axmann, H. Hofmann, and A. Petri-Fink, "Optical properties of annealed $\mathrm{Mn}^{2+}$-doped $\mathrm{ZnS}$ nanoparticles," Journal of Luminescence, vol. 128, no. 1, pp. 92-98, 2008.

[4] P. V. Radovanovic, C. J. Barrelet, S. Gradecak, F. Qian, and C. M. Lieber, "General synthesis of manganese-doped II-VI and III-V semiconductor nanowires," Nano Letters, vol. 5, no. 7, pp. 1407-1411, 2005.

[5] P. Lommens, F. Loncke, P. F. Smet et al., "Dopant incorporation in colloidal quantum dots: a case study on $\mathrm{Co}^{2+}$ doped ZnO," Chemistry of Materials, vol. 19, no. 23, pp. 5576-5583, 2007.

[6] D. -L. Hou, X. -J. Ye, H. -J. Meng et al., "Magnetic properties of Mn-doped $\mathrm{ZnO}$ powder and thin films," Materials Science and Engineering B, vol. 138, no. 2, pp. 184-188, 2007.

[7] R. Tu, B. Liu, Z. Wang et al., "Amine-capped $\mathrm{ZnS}-\mathrm{Mn}^{2+}$ nanocrystals for fluorescence detection of trace TNT explosive," Analytical Chemistry, vol. 80, no. 9, pp. 3458-3465, 2008.

[8] N. Pradhan, D. Goorskey, J. Thessing, and X. Peng, "An alternative of CdSe nanocrystal emitters: pure and tunable impurity emissions in ZnSe nanocrystals," Journal of the American Chemical Society, vol. 127, no. 50, pp. 17586-17587, 2005.

[9] L. Zu, D. J. Norris, T. A. Kennedy, S. C. Erwin, and A. L. Efros, "Impact of ripening on manganese-doped ZnSe nanocrystals," Nano Letters, vol. 6, no. 2, pp. 334-340, 2006.

[10] B. Xi, D. Xu, S. Xiong et al., "Preparation and characterization of cubic and hexagonal polytypes of $\mathrm{ZnSe}: \mathrm{Cu}^{2+}$ onedimensional nanostructures," Journal of Physical Chemistry C, vol. 112, no. 14, pp. 5333-5338, 2008.
[11] N. Pradhan and X. Peng, "Efficient and color-tunable Mndoped ZnSe nanocrystal emitters: control of optical performance via greener synthetic chemistry," Journal of the American Chemical Society, vol. 129, no. 11, pp. 3339-3347, 2007.

[12] N. S. Norberg, G. L. Parks, G. M. Salley, and D. R. Gamelin, "Giant excitonic zeeman splittings in colloidal $\mathrm{Co}^{2+}$-doped ZnSe quantum dots," Journal of the American Chemical Society, vol. 128, no. 40, pp. 13195-13203, 2006.

[13] W. C. W. Chan and S. Nie, "Quantum dot bioconjugates for ultrasensitive nonisotopic detection," Science, vol. 281, no. 5385, pp. 2016-2018, 1998.

[14] N. Murase and M. Gao, "Preparation and photoluminescence of water-dispersible ZnSe nanocrystals," Materials Letters, vol. 58, no. 30, pp. 3898-3902, 2004.

[15] N. O. Dantas, L. Damigo, F. Qu et al., "Raman investigation of $\mathrm{ZnO}$ and $\mathrm{Zn1-xMnxO}$ nanocrystals synthesized by precipitation method," Journal of Non-Crystalline Solids, vol. 354, no. 42-44, pp. 4827-4829, 2008.

[16] K. Palaniappan, C. Xue, G. Arumugam, S. A. Hackney, and J. Liu, "Water-soluble, cyclodextrin-modified CdSe-CdS coreshell structured quantum dots," Chemistry of Materials, vol. 18, no. 5, pp. 1275-1280, 2006.

[17] P. T. K. Chin, J. W. Stouwdam, and R. A. J. Janssen, "Highly luminescent ultranarrow Mn doped ZnSe nanowires," Nano Letters, vol. 9, no. 2, pp. 745-750, 2009.

[18] S. Mahamuni, A. D. Lad, and S. Patole, "Photoluminescence properties of manganese-doped zinc selenide quantum dots," Journal of Physical Chemistry C, vol. 112, no. 7, pp. 2271-2277, 2008.

[19] T. J. Norman Jr., D. Magana, T. Wilson et al., "Optical and surface structural properties of $\mathrm{Mn}^{2+}$-doped $\mathrm{ZnSe}$ nanoparticles," Journal of Physical Chemistry B, vol. 107, no. 26, pp. 6309-6317, 2003.

[20] Y. Zhang, C. Gan, J. Muhammad, D. Battaglia, X. Peng, and M. Xiao, "Enhanced fluorescence intermittency in Mn-doped single ZnSe quantum dots," Journal of Physical Chemistry C, vol. 112, no. 51, pp. 20200-20205, 2008. 

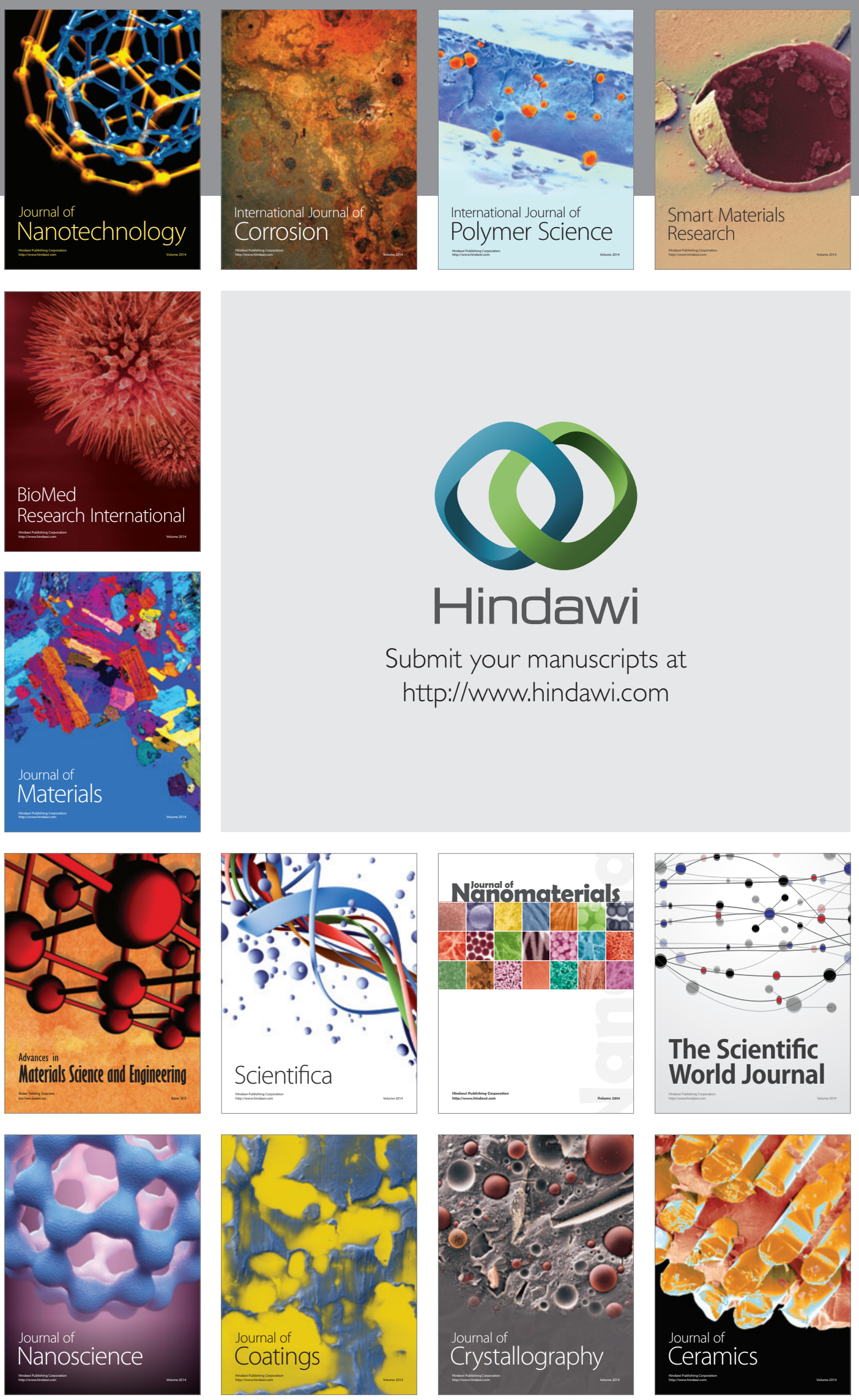

The Scientific World Journal

Submit your manuscripts at

http://www.hindawi.com

\section{World Journal}

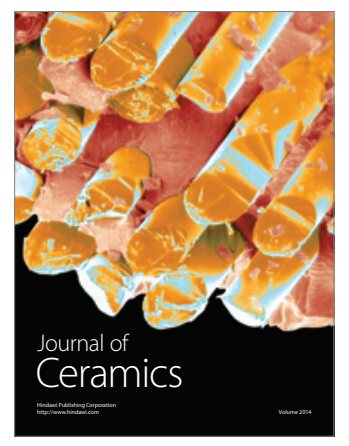

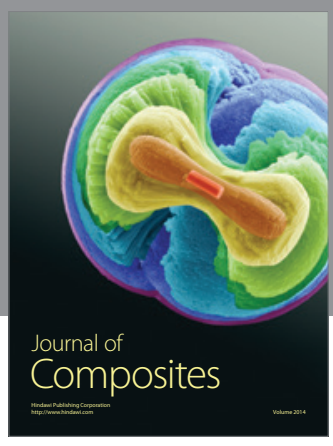
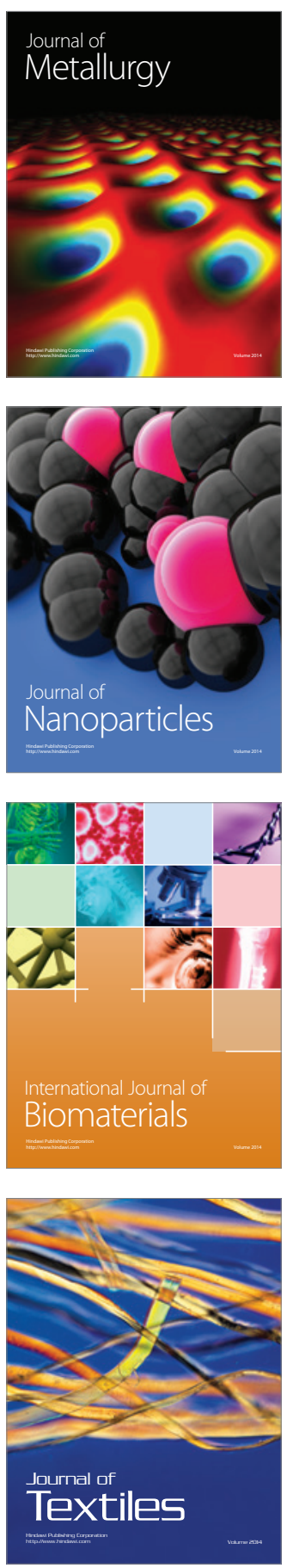